\title{
THE LERAY SPECTRAL SEQUENCE IS MOTIVIC
}

\author{
DONU ARAPURA
}

Our goal is to prove that the Leray spectral sequence associated to a map of algebraic varieties is motivic in the following sense: If the singular cohomology groups of the category of quasiprojective varieties defined over a subfield of $\mathbb{C}$ can be canonically endowed with some additional structure, such as its mixed Hodge structure or its motive in Nori's sense. Then the Leray spectral sequence for a projective map in this category is automatically compatible with this structure. Establishing the compatibility of Leray with mixed Hodge structures was our primary objective, and there were some important precedents for this. Zucker [Z] established compatibility for a map to a curve. This was proved following a delicate analysis of variations of Hodge structure on curves and their cohomology. Saito, in his rather formidable work on (mixed) Hodge modules [S1, S2], addressed the compatibility issue in general, albeit somewhat indirectly. His theory mirrors the theory of perverse $l$-adic sheaves $\mathrm{BBD}$. Among other things, Saito obtained an analogue of the decomposition theorem, which gives, in practice, a replacement for the Leray spectral sequence. In view of this history, it came as a pleasant surprise to author that one could get quite far by using nothing beyond the standard functoriality properties established by Deligne D2.

A very brief outline of the proof of the main result (theorem 3.1) is as follows. We first show that the spectral sequence associated to a filtration by subvarieties is motivic. Then we identify the Leray spectral sequence with such a spectral sequence associated to a suitable filtration. The quasiprojectivity assumption is imposed mainly for convenience since it permits considerable simplifications of the arguments. When the map is smooth and the coefficients are rational, the Leray spectral sequence degenerates, and we can exhibit its motivic structure in a more elementary fashion by theorem 2.1. This special case is already sufficient for many purposes.

As an antidote to all these generalities, we conclude the paper with a simple geometric example to illustrate how these results can be applied (some readers may wish to start with this). These results can be used to simplify or justify arguments that have already appeared in the literature (e.g. AS, 6.3.1], L, 3.4]). Another byproduct is a relatively cheap construction of a mixed Hodge structure on the cohomology of variation of Hodge structure of geometric origin. This can be used as an alternative to Saito's machinery for certain applications (e.g. [H] ). We have not checked compatibility with Saito's construction, but we expect that it should be true.

I would like to express my warmest thanks to Madhav Nori for sharing his insight into these problems with me. The special case (2.1) was found by him independently, and the proof of the general case (3.1) was based on his observation that it is possible to construct "cellular decompositions" for affine varieties with

Author partially supported by the NSF. 
respect to constructible sheaves. I would also like to thank Chris Peters for pointing out a gap in the original proof of theorem 2.1] and for suggesting a remedy; I will say more about that below. My thanks also to the referee for suggesting some improvements in the exposition.

\section{Preliminaries}

Let us fix some conventions and terminology. We will drop indices and other decorations from the notation whenever they seem unnecessary. Let $V_{\kappa}$ be the category of quasiprojective schemes over be a field $\kappa$. We refer to these objects as varieties whether not they are irreducible. The dimension is the dimension of the largest component. Let $V_{\kappa}^{2}$ be the category whose objects are pairs $(X, Y)$ with $Y \subseteq X$ closed. A morphism from $(X, Y) \rightarrow\left(X^{\prime}, Y^{\prime}\right)$ is a morphism of $\kappa$-schemes $X \rightarrow X^{\prime}$ such that $f(Y) \subseteq Y^{\prime}$. The category $V_{\kappa}^{3}$ of triples of closed embeddings $Z \subseteq Y \subseteq X$ in $V_{\kappa}$ can be defined in a similar fashion. We define a weak cohomology theory over $\kappa$ to be a functor $\mathcal{H}: V_{\kappa}^{2} \rightarrow \mathcal{A}$ to an Abelian category such that for any object $Z \subseteq Y \subseteq X$ in $V_{\kappa}^{3}$, there are natural transformations

$$
\mathcal{H}^{i}(Y, Z) \rightarrow \mathcal{H}^{i+1}(X, Y)
$$

which fit into a long exact sequence:

$$
\ldots \mathcal{H}^{i}(Y, Z) \rightarrow \mathcal{H}^{i+1}(X, Y) \rightarrow \mathcal{H}^{i+1}(X, Z) \ldots
$$

As usual, we write $\mathcal{H}^{i}(X)=\mathcal{H}^{i}(X, \emptyset)$. $\mathcal{A}$ will be called the coefficient category.

Here is a list of examples.

(Betti) Let $\kappa=\mathbb{C}$, then take $\mathcal{H}=H$ to be singular (or Betti) cohomology with $\mathbb{Z}$ coefficients. In this example the coefficient category is the category of Abelian groups $A b$

(MHS) Let $\kappa=\mathbb{C}$, take $\mathcal{H}=H_{M H S}$ as $H$ together with its canonical mixed Hodge structure D2. The coefficient category is the category of mixed Hodge structures.

( $\sigma$-Betti) Let $\sigma: \kappa \hookrightarrow \mathbb{C}$ be an embedding. The composition $H_{\sigma}$ of $H$ with base extension by $\sigma$ gives a weak theory with coefficients $A b$. one can define $H_{\sigma, M H S}$ in a similar fashion.

(l-adic) Let $l$ be a prime different from the characteristic of $\kappa$, let $\bar{\kappa}$ denote the separable closure and let $\mathcal{H}_{l}^{i}(X, Y)=H_{e t}^{i}\left(X \otimes \bar{\kappa}, j_{Y, X !} \mathbb{Q}_{l}\right)$ where $j_{X, Y}$ : $X-Y \hookrightarrow X$ is the inclusion. The long exact sequence (11) is induced from

$$
0 \rightarrow j_{X, Y !} \mathbb{Q}_{l} \rightarrow j_{X, Z !} \mathbb{Q}_{l} \rightarrow j_{Y, Z !} \mathbb{Q}_{l} \rightarrow 0
$$

$\mathcal{A}$ is the category of $l$-adic representations of $\operatorname{Gal}(\bar{\kappa} / \kappa)$.

(AH) When $\kappa$ is embeddable into $\mathbb{C}$, Jannsen [J] 6.11.1] has essentially constructed a weak cohomology theory $H_{A H}$ with values in his category of integral mixed realizations $M R_{k}$. Here we ignore Tate twists. The cohomology of a pair can defined by combining Jannsen's methods with the simplicial mapping cone construction [D2, 6.3].

(ECM) When $\kappa$ is embeddable into $\mathbb{C}$, Nori (unpublished) has constructed the universal weak cohomology theory $H_{E C M}$. The coefficient category is his category of effective cohomological motives.

We assume from now on that $\kappa$ is embeddable into $\mathbb{C}$. Fix one such embedding $\sigma: \kappa \hookrightarrow \mathbb{C}$. We will call a weak cohomology theory $\mathcal{H}: V_{\kappa}^{2} \rightarrow \mathcal{A}$ an enriched (rational) $\sigma$-Betti theory if there exist a faithful exact functor $\Phi: \mathcal{A} \rightarrow A b$ ( 
$\Phi: \mathcal{A} \rightarrow \mathbb{Q}$-(vector spaces)) such that $\left.H_{\sigma}=\Phi \circ \mathcal{H}\left(H_{\sigma} \otimes \mathbb{Q}=\Phi \circ \mathcal{H}\right)\right)$. Both $H_{\sigma, M H S}$ with the obvious $\Phi$, and $H_{A H}$ and $H_{E C M}$ provided examples of enriched Betti theories.

By a spectral sequence in an Abelian category $\mathcal{A}$, we mean a collection of bigraded objects $E_{r}^{\bullet \bullet}$ with differentials of bidegree $(r,-r+1)$, for $r \geq r_{0}$ for some $r_{0} \geq 0$, together with a filtered graded object $\left(H^{\bullet}, F^{\bullet}\right)$ with an isomorphisms

$$
E_{r+1} \cong H^{\bullet}\left(E_{r}\right), E_{\infty} \cong G r_{F} H .
$$

We will denote the whole thing by $E_{r_{0}} \Rightarrow H$ or simply $E_{r_{0}}$. Clearly the set of spectral sequences (for fixed $r_{0}$ ) form a category $S S\left(\mathcal{A}, r_{0}\right)$ in its own right. Given an exact functor of Abelian categories $\Phi: \mathcal{A} \rightarrow \mathcal{B}$ and a spectral sequence $E_{r_{0}}$ in $\mathcal{B}$, by a lift to $\mathcal{A}$ we mean a spectral sequence $\mathcal{E}_{r_{0}}$ in $\mathcal{A}$ and an isomorphism $E_{r_{0}} \cong \Phi\left(\mathcal{E}_{r_{0}}\right)$. More generally, given a functor $F: C \rightarrow S S\left(\mathcal{B}, r_{0}\right)$, a natural lift is a functor $F^{\prime}: C \rightarrow S S\left(\mathcal{A}, r_{0}\right)$ and a natural isomorphism $\Phi \circ F^{\prime} \cong F$.

Given a continuous map of topological spaces $f: X \rightarrow Y$ and sheaf $\mathcal{F}$ on $X$, the Leray spectral sequence

$$
\begin{gathered}
\mathcal{L}_{2}^{p, q}(f, \mathcal{F})=H^{p}\left(Y, R^{q} f_{*} \mathcal{F}\right) \\
\mathcal{L}_{\infty}^{p, q}=\operatorname{Gr}_{L}^{p} H^{p+q}(X, \mathcal{F})
\end{gathered}
$$

provides the basic example of a spectral sequence in $A b$. The Leray filtration is given by

$$
L^{p} H^{i}(X, \mathcal{F})=\operatorname{image}\left[H^{i}\left(Y, \tau_{\leq i-p} \mathbb{R} f_{*} \mathcal{F}\right) \rightarrow H^{i}\left(Y, \mathbb{R} f_{*} \mathcal{F}\right) \cong H^{i}(X, \mathcal{F})\right] .
$$

This is functorial in the sense that a commutative diagram

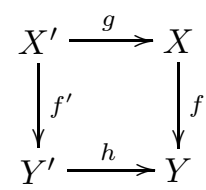

induces morphism

$$
\mathcal{L}_{2}^{p q}(f, \mathcal{F}) \rightarrow \mathcal{L}_{2}^{p q}\left(f^{\prime}, g^{*} \mathcal{F}\right) .
$$

Functoriallity follows from the construction which we briefly recall along with some related homological algebra. A biregular filtered complex $\left(K^{\bullet}, F^{\bullet}\right)$ in an Abelian category gives rise to a spectral sequence

$$
E_{1}^{p q}(K, F)=H^{p+q}\left(G r_{F}^{p} K^{\bullet}\right) \Rightarrow H^{p+q}\left(K^{\bullet}\right)
$$

This can be constructed using explicit formulas or in terms of exact couples which we recall later. The shifted filtration ("filtration decalé") [D2, 1.3.3] is

$$
\operatorname{Dec}(F)^{p} K^{n}=\left\{\alpha \in F^{p+n} K^{n} \mid d \alpha \in F^{p+n+1}\right\} .
$$

For example, the canonical filtration $\tau^{\bullet}=\tau_{\leq-}$• is just $\operatorname{Dec}(G)$, where $G$ is the trivial filtration $G^{0}=K, G^{1}=0$. The spectral sequence with respect to the shifted and unshifted filtrations are the same up to a change of indices [D2 1.3.4]:

$$
E_{r}^{p q}(K, \operatorname{Dec}(F)) \cong E_{r+1}^{2 p+q,-p}(K, F)
$$

A morphism of filtered complexes is a filtered quasiisomorphism if it induces an isomorphism on the associated graded complexes. Such a morphism induces an isomorphism of (44). The filtered derived category [D2 7.1, 1.4] is obtained from the category of filtered complexes by inverting filtered quasiisomorphisms; 
the spectral sequence (4) extends to this setting. Any object $\mathcal{F}$ of the derived category, gives rise to a well defined object $(\mathcal{F}, \tau)$ of the filtered derived category. A filtered acyclic resolution is a filtered quasiisomorphism to a filtered complex whose associated graded is acyclic. Derived functors, which exist in the filtered context, are computed by applying the functor to a filtered acyclic resolution of the original complex. In particular, given a filtered complex of sheaves $\left(K^{\bullet}, F^{\bullet}\right)$, we have an object $\mathbb{R} \Gamma(K, F)$ of the filtered derived category of Abelian groups, and this maps to $\mathbb{R} \Gamma(K)$ under the forgetful functor to the ordinary derived category. There is a spectral sequence

$$
E_{1}^{p q}(\mathbb{R} \Gamma(K, F))=\mathbb{H}^{p+q}\left(G r_{F}^{p} K^{\bullet}\right) \Rightarrow \mathbb{H}^{p+q}\left(K^{\bullet}\right)
$$

Suppose $f: X \rightarrow Y$ is a continuous map, and $\mathcal{F}$ a sheaf on $X$. The canonical filtration on $\mathbb{R} f_{*} \mathcal{F}$ induces the Leray spectral sequence with a shift of indices D2, 1.4.8]:

$$
E_{r}^{p q}\left(\mathbb{R} \Gamma\left(\mathbb{R} f_{*} \mathcal{F}, \tau\right)\right) \cong \mathcal{L}_{r+1}^{2 p+q,-p}(f, \mathcal{F})
$$

\section{Smooth Projective Maps}

For the remainder of this paper, we will be concerned with varieties defined over a subfield $\sigma: \kappa \hookrightarrow \mathbb{C}$. By a sheaf on a such variety $X$, we will mean a sheaf on $\left(X \times_{\sigma} \text { Spec } \mathbb{C}\right)^{a n}$. We write $H^{i}(X, \mathcal{F})$ instead of $H^{i}\left(\left(X \times_{\sigma} \text { Spec } \mathbb{C}\right)^{a n}, \mathcal{F}\right)$. We say that a sheaf $\mathcal{F}$ of $\mathbb{Z}$-modules on $X$ is weakly constructible if there is a partition of $X$ into Zariski locally closed sets $\mathcal{Z}$ defined over $\kappa$ such that $\left.\mathcal{F}\right|_{Z}$ is locally constant for each $Z \in \mathcal{Z} ; \mathcal{F}$ is constructible if in addition the stalks are finitely generated.

Theorem 2.1. Let $Y$ be a smooth complex irreducible quasiprojective variety. Then for each $p$, there a morphism $Y_{p} \rightarrow Y$ with the following property: If $f: X \rightarrow Y$ is a smooth projective morphism, then

$$
L^{p} H^{i}(X, \mathbb{Q})=\operatorname{ker}\left[H^{i}(X, \mathbb{Q}) \rightarrow H^{i}\left(X_{p}, \mathbb{Q}\right)\right]
$$

where $X_{p}=f^{-1} Y_{p}$. There are morphisms $Y_{p-1} \rightarrow Y_{p}$ over $Y$ compatible with inclusions $L^{p} \subseteq L^{p-1}$. If $Y$ is defined over an algebraically closed subfield $\kappa \subseteq \mathbb{C}$, then $Y_{p}$ etcetera can be defined over $\kappa$.

Remark 2.2. In an earlier incarnation of this paper, we stated and attempted to prove this theorem without assuming smoothness of $Y$. However, C. Peters pointed a gap in the original form of lemma [2.5, and suggested a fix using work of [HL] among things; $H$ would have to be allowed to be replaced by a multiple. In the interests of simplicity, we opted to state and prove this under a more restrictive hypotheses. In any case, this theorem is superceded by theorem [3.1.

Lemma 2.3 (Jouanolou). Let $Y$ be a quasiprojective variety over a field $\kappa$. There exists an affine $\kappa$-variety $Y^{\prime}$ and a morphism $Y^{\prime} \rightarrow Y$ where the fibers are isomorphic to an affine space.

Proof. See [Jo 1.5]

Call a $\kappa$-variety $F$ contractible, if $(F \times \mathbb{C})^{a n}$ is contractible. 
Corollary 2.4. Let $X \rightarrow Y$ be a morphism of quasiprojective varieties over $\kappa$. There exists a commutative diagram

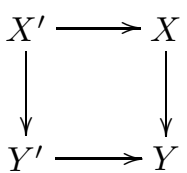

where both $X^{\prime}$ and $Y^{\prime}$ are affine, and the fibers of the horizontal maps are contractible.

Proof. Apply the lemma to $Y$ to obtain $Y^{\prime}$, and then again to $X \times_{Y} Y^{\prime}$ to obtain $X^{\prime}$.

We need the following version of the weak Lefschetz theorem.

Lemma 2.5. Let $L$ be a locally constant sheaf on an $n$ dimensional nonsingular complex affine variety $Y$. Then $H^{i}(Y, L)=0$ for $i>n$, and $H^{i}(Y, L) \rightarrow H^{i}(H, L)$ is injective for $i<n$ and any general affine hyperplane section $H$.

Proof. The vanishing follows from the topological analogue of Artin's theorem about the vanishing of the cohomology of constructible sheaves on $Y$ in degrees $>n$. (A proof can be found in GM. One can also deduce this from lemma 3.5 and induction.) For the second statement, we apply [AS, thm 6.1.1]

Proof of theorem [2.1. We first treat the special case where $Y$ is affine, and prove this by induction on dimension. The initial case where $Y$ is a point, or more generally a union of points, is vacuously true. We can take $Y_{0}=\emptyset$. Assume that $\operatorname{dim} Y=n>0$. Choose a general hyperplane section $H$ (defined over $\kappa$ ), and consider the cartesian square

$$
\begin{gathered}
X_{H} \rightarrow \quad X \\
g \downarrow \\
H \rightarrow \\
H \quad Y
\end{gathered}
$$

This induces a morphism of Leray spectral sequences

$$
\mathcal{L}_{2}^{p q}(f, \mathbb{Q}) \rightarrow \mathcal{L}_{2}^{p q}(g, \mathbb{Q})
$$

Furthermore, by [1], both spectral sequences degenerate at $E_{2}$. Together with lemma 2.5 this implies that $\mathcal{L}_{\infty}^{p q}(f, \mathbb{Q})$ and $\mathcal{L}_{\infty}^{p q}(g, \mathbb{Q})$ vanish for $p>n$ and $p>n-1$ respectively. Moreover, the map

$$
\mathcal{L}_{\infty}^{p q}(f, \mathbb{Q}) \rightarrow \mathcal{L}_{\infty}^{p q}(g, \mathbb{Q})
$$

is an injective if $p<n$. Thus the smallest step of the Leray filtration

$$
L^{n} H^{i}(X, \mathbb{Q})=\mathcal{L}_{\infty}^{n, i-n}(f, \mathbb{Q})=\operatorname{ker}\left[H^{i}(X, \mathbb{Q}) \rightarrow H^{i}\left(X_{H}, \mathbb{Q}\right)\right]
$$

Therefore, we can take $Y_{n}=H$. Suppose that $p<n$. By induction, there is a morphism of varieties $H_{p} \rightarrow H$ such that

$$
L^{p} H^{i}\left(X_{H}, \mathbb{Q}\right)=\operatorname{ker}\left[H^{i}\left(X_{H}, \mathbb{Q}\right) \rightarrow H^{i}\left(f^{-1} H_{p}, \mathbb{Q}\right)\right]
$$

Since $L^{p}$ is compatible with the restriction $H^{i}(X, \mathbb{Q}) \rightarrow H^{i}\left(X_{H}, \mathbb{Q}\right)$, it follows that

$$
L^{p} H^{i}(X, \mathbb{Q}) \subseteq \operatorname{ker}\left[H^{i}(X, \mathbb{Q}) \rightarrow H^{i}\left(f^{-1} H_{p}, \mathbb{Q}\right)\right]
$$

We claim equality holds. Suppose to the contrary, that there exists $\alpha$ in the complement of the right and left hand sides. Choose the least $q$ such that $\alpha \in L^{q} H^{i}(X, \mathbb{Q})$. 
We have $q<p$. Then the class $G r_{L}^{q}(\alpha) \neq 0$, which implies that $G r_{L}^{q}\left(\left.\alpha\right|_{X_{H}}\right) \neq 0$. Therefore $\left.\alpha\right|_{X_{H}} \notin L^{p}$. It follows that its restriction to $f^{-1} H_{p}$ is nonzero, and this is a contradiction. This proves the claim. Setting $Y_{p}=H_{p}$ completes the proof for affine $Y$.

Let $Y^{\prime} \rightarrow Y$ be as in lemma 2.3 and $X^{\prime}=X \times_{Y} Y^{\prime}$. Since the fibers of $Y^{\prime} \rightarrow Y$ are contractible, the maps $f^{a n}$ and $f^{\prime a n}: X^{\prime a n} \rightarrow Y^{\prime a n}$ are homotopy equivalent. Thus there is an isomorphism $H^{*}(X) \cong H^{*}\left(X^{\prime}\right)$ strictly compatible with Leray filtrations, i.e. that the induced map on $G r_{L}$ is an isomorphism. By the previous case, there exists $Y_{p}^{\prime} \rightarrow Y^{\prime}$ such that $L^{p} H^{i}\left(X^{\prime}\right)$ is the kernel of the map to $H^{i}\left(f^{\prime-1} Y_{p}^{\prime}\right)$. Then the composition $Y_{p}=Y_{p}^{\prime} \rightarrow Y$ will have the required property.

Corollary 2.6. The Leray filtration on $H^{i}(X, \mathbb{Q})$ is a filtration by sub-mixed Hodge structures. More generally, for any enriched rational $\sigma$-Betti theory $\left(\mathcal{H}: V_{\kappa}^{2} \rightarrow\right.$ $\mathcal{A}, \Phi)$, the Leray filtration on $H^{i}(X, \mathbb{Q})$ is the image of a filtration on $\mathcal{H}^{i}(X)$.

\section{MAIN THEOREM}

Fix an embedding $\sigma: \kappa \hookrightarrow \mathbb{C}$ and an enriched $\sigma$-Betti theory $\left(\mathcal{H}: V_{\kappa}^{2} \rightarrow \mathcal{A}, \Phi\right)$. Let $f: X \rightarrow Y$ be a projective morphism of quasiprojective varieties over $\kappa$, then we can form the Leray spectral sequence

$$
E_{2}=H_{\sigma}^{p}\left(Y, R^{q} f_{\sigma *} \mathbb{Z}\right) \Rightarrow H_{\sigma}^{p+q}(X, \mathbb{Z})
$$

where $f_{\sigma}$ is the analytic map associated to $f \times_{\sigma}$ Spec $\mathbb{C}$. As explained in section 1 , a natural lift of (9) consists of a spectral sequence

$$
\mathcal{E}_{2}^{p q}(f) \Rightarrow \mathcal{H}
$$

in $\mathcal{A}$ which is compatible with base change (3) and maps to (9) under $\Phi$.

Theorem 3.1. The Leray spectral sequence has a natural lift to $\mathcal{A}$. (Thus it is motivic.)

Corollary 3.2. The Leray spectral sequence of a projective map of complex quasiprojective varieties can be lifted naturally to the category of mixed Hodge structures.

Since the proof of the theorem will be spread out over a long series of lemmas, it may be worthwhile to outline the main steps. (We suppress $\sigma$ in the outline.)

A) If $X$ has an increasing filtration $X \bullet$ by closed sets, then lemma 3.8 will yield a spectral sequence of Abelian groups

$$
E_{1}^{p q}\left(X_{\bullet}, \mathbb{Z}\right) \Rightarrow H^{p+q}(X, \mathbb{Z})
$$

which is motivic in the sense that it has a natural lift to $\mathcal{A}$.

B) If $X_{\bullet}$ is given by pulling back a filtration $Y_{\bullet}$ from $Y$, then in lemma 3.13 we will construct a morphism

$$
H^{p}\left(Y, R^{q} f_{*} \mathbb{Z}\right) \rightarrow E_{2}^{p q}\left(X_{\bullet}, \mathbb{Z}\right)
$$

from the Leray spectral sequence to the one above from $E_{2}$ onwards. Note that this morphism is given in the category of Abelian groups; the left side has no motivic structure a priori.

C) As in the proof of theorem 2.1 we can reduce to the case where $Y$ is affine. Then by applying lemma 3.7 to the direct images $R^{q} f_{*} \mathbb{Z}$, we can choose the $Y_{\bullet}$ so that the morphism of step $\mathrm{B}$ is an isomorphism. This gives the desired motivic structure on Leray. 
D) The final step is to check independence of choices and naturality.

It is worth remarking that there certain parallels between this proof and the proof of theorem 2.1 For example, the collection of varieties $\left\{Y_{\bullet}\right\}$ play essentially the same role in both proofs.

In order to flesh out the above sketch, we need some more notation. We can form a category $F V_{\kappa}$ as follows. The objects are pairs consisting of a variety $X$ in $V_{\kappa}$ and an increasing exhaustive filtration

$$
X=\ldots X_{M+1}=X_{M} \supseteq X_{M-1} \supseteq \ldots X_{-1}=\emptyset
$$

by closed sets. A morphism of $f:\left(X, X_{\bullet}\right) \rightarrow\left(Y, Y_{\bullet}\right)$ is a morphism of varieties such that $f\left(X_{i}\right) \subseteq Y_{i}$. Suppose that $\left(Y, Y_{\bullet}\right)$ is an object of $F V_{\kappa}$. Let $Y_{a}^{o}=Y_{a}-Y_{a-1}$, and let $j_{a}: Y_{a}^{o} \hookrightarrow Y_{a}, k_{a}: Y-Y_{a} \rightarrow Y$ and $i_{a}: Y_{a} \rightarrow Y$ denote the inclusions. Fix a sheaf $\mathcal{F}$ on $Y$, and let $\mathcal{F}_{a}=\left.j_{a !} \mathcal{F}\right|_{Y_{a}^{o}}, S^{a}\left(Y_{\bullet}, \mathcal{F}\right)=k_{(a-1) !} k_{a-1}^{*} \mathcal{F}, S^{0}\left(Y_{\bullet}, \mathcal{F}\right)=\mathcal{F}$. We will suppress one or both arguments of $S$ if these are clear from context. We have a filtration by "skeleta": $\mathcal{F}=S^{0}(\mathcal{F}) \supset S^{1}(\mathcal{F}) \supset \ldots$ such that $S^{a} / S^{a+1} \cong i_{a *} \mathcal{F}_{a}$. This isomorphism follows from the snake lemma applied to the diagram

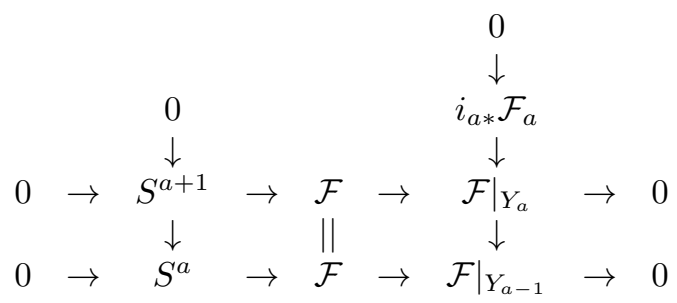

Given a complex of sheaves of Abelian groups $\mathcal{F}^{\bullet}, S^{\bullet}\left(Y_{\bullet}, \mathcal{F}^{\bullet}\right)$ is a filtration by subcomplexes. The spectral sequence (7]) for $\left(\mathcal{F}^{\bullet}, S\left(Y_{\bullet}, \mathcal{F}^{\bullet}\right)\right)$ becomes

$$
E_{1}^{a b}\left(Y_{\bullet}, \mathcal{F}^{\bullet}\right)=\mathbb{H}^{a+b}\left(Y, i_{a *} \mathcal{F}_{a}^{\bullet}\right) \Rightarrow \mathbb{H}^{a+b}\left(Y, \mathcal{F}^{\bullet}\right)
$$

The differential

$$
d_{1}: E_{1}^{a, b}\left(Y_{\bullet}, \mathcal{F}^{\bullet}\right) \rightarrow E_{1}^{a+1, b}\left(Y_{\bullet}, \mathcal{F}^{\bullet}\right)
$$

is given explicitly as a composition of

$$
\mathbb{H}^{a+b}\left(Y, \mathcal{F}_{a}^{\bullet}\right) \rightarrow \mathbb{H}^{a+b}\left(Y,\left.\mathcal{F}^{\bullet}\right|_{Y_{a}}\right)
$$

and the connecting map

$$
\mathbb{H}^{a+b}\left(Y,\left.\mathcal{F}^{\bullet}\right|_{Y_{a}}\right) \rightarrow \mathbb{H}^{a+b+1}\left(Y,\left.\mathcal{F}^{\bullet}\right|_{Y_{a+1}}\right)
$$

Given a morphism $f$ and a complex $\mathcal{F}^{\bullet}$ on $Y$, we get a morphism of spectral sequences

$$
f^{*}: E_{1}^{a b}\left(Y_{\bullet}, \mathcal{F}^{\bullet}\right) \rightarrow E_{1}^{a b}\left(X_{\bullet}, f^{*} \mathcal{F}^{\bullet}\right) .
$$

In particular,

$$
\left(Y, Y_{\bullet}\right) \mapsto E_{1}^{a b}\left(Y_{\bullet}, \mathbb{Z}\right)
$$

is a functor from $F V_{\kappa} \rightarrow S S(A b, 1)$

Since the functors $G r_{S^{a}}=\left(i_{a} \circ j_{a}\right) ! j_{a}^{*}$ are exact, a quasiisomorphism $\mathcal{F}^{\bullet} \rightarrow \mathcal{G}^{\bullet}$ gives rise to a filtered quasiisomorphism $\left(\mathcal{F}^{\bullet}, S^{\bullet}\left(\mathcal{F}^{\bullet}\right)\right) \rightarrow\left(\mathcal{G}^{\bullet}, S^{\bullet}\left(\mathcal{G}^{\bullet}\right)\right)$. Thus an object $\mathcal{F}$ in the derived category $D^{+}(Y)$ gives rise to well defined object $\left(\mathcal{F}, S^{\bullet}(\mathcal{F})\right)$ in the filtered derived category $D F^{+}(Y)$. If $\mathcal{F}$ is replaced by an injective resolution $\mathcal{I}^{\bullet}$, then this is represented by $\left(\mathcal{I}^{\bullet}, S^{\bullet}\left(\mathcal{I}^{\bullet}\right)\right)$ thanks to:

Lemma 3.3. If $\mathcal{I}$ is injective then $G r_{S^{a}} \mathcal{I}$ and $S^{\bullet}\left(Y_{\bullet}, \mathcal{I}\right)$ are flasque. 
Proof. Both $j^{*}$ and $j$ ! preserve injectivity for any open immersion $j$. Injective sheaves are flasque, and $i_{*}$ takes flasque sheaves to flasque sheaves for any closed immersion $i$. This proves that $G r_{S^{a}} \mathcal{I}$ is flasque. Since the class of flasque sheaves is closed under extensions, $S^{\bullet}\left(Y_{\bullet}, \mathcal{I}\right)$ is also flasque.

We say that an object $\left(Y, Y_{\bullet}\right)$ is cellular with respect to a sheaf $\mathcal{F}$ if $H^{i}\left(Y, \mathcal{F}_{a}\right)=$ 0 , for $i \neq a$. The terminology is suggested by the analogy with $C W$ or cell complexes in topology with its filtration by skeleta.

Lemma 3.4. Suppose that $H^{i}\left(Y, \mathcal{F}_{a}\right)=0$ for $i \neq a$, then

$$
H^{i}(Y, \mathcal{F}) \cong H^{i}\left(E_{1}^{\bullet 0}\left(Y_{\bullet}, \mathcal{F}\right), d_{1}\right)
$$

Proof. This follows immediately from the spectral sequence (10).

Lemma 3.5 (Beilinson). If $\mathcal{F}$ is a weakly constructible sheaf on an $n$ dimensional affine variety $Y$, there exists a nonempty open set $j: U \hookrightarrow X$ such that

$$
H^{i}\left(Y, j ! j^{*} \mathcal{F}\right)=0
$$

unless $i=n$.

Proof. See $[\mathbb{N}]$.

Corollary 3.6. Suppose that $\mathcal{F}_{1}, \mathcal{F}_{2}, \ldots$ is a finite collection of weakly constructible sheaves on $Y$ and that $Y^{\prime} \subset Y$ is a proper closed set. There exists a nonempty open set $j: U \hookrightarrow X-Y^{\prime}$ such that for all $k$,

$$
H^{i}\left(Y, j_{!} j^{*} \mathcal{F}_{k}\right)=0
$$

unless $i=n$.

Proof. Apply the lemma to $\left(V, \mathcal{F}_{1} \oplus \mathcal{F}_{2} \oplus \ldots\right)$, where $V$ is an affine subset of $X-Y^{\prime}$,

The existence of cellular filtrations is given by:

Lemma 3.7. Let $Y$ be an $n$ dimensional affine variety. Suppose that $\mathcal{F}, \ldots$ is a finite collection of weakly constructible sheaves on $Y$ and that

$$
Y_{0}^{\prime} \subset Y_{1}^{\prime} \subset \ldots Y_{n}^{\prime}=Y
$$

is a chain of closed sets such $\operatorname{dim} Y_{i}^{\prime}=i$. There exists a filtration

$$
Y_{0} \subset Y_{1} \subset \ldots Y_{n}=Y
$$

such that

(1) $\operatorname{dim} Y_{i}=i$.

(2) $Y_{i}^{\prime} \subseteq Y_{i}$.

(3) $Y_{\bullet}$ is cellular with respect to $\mathcal{F}, \ldots$

Proof. This follows from corollary 3.6 and induction on $\operatorname{dim} Y$.

Lemma 3.8. For any pair $\left(X, X_{\bullet}\right)$ in $F V_{\kappa}$,

$$
E_{1}^{p q}\left(X_{\bullet}, \mathbb{Z}\right) \Rightarrow H^{p+q}(X, \mathbb{Z})
$$

can be lifted naturally to $\mathcal{A}$. 
Proof. The spectral sequence $E_{1}\left(X_{\bullet}, \mathbb{Z}\right)$ can be described using the language of exact couples [W, 5.9]. We set

$$
D_{1}=\bigoplus H^{\bullet}\left(X, S^{\bullet}(\mathbb{Z})\right)=\bigoplus H^{\bullet}\left(X, X_{\bullet} ; \mathbb{Z}\right)
$$

and

$$
E_{1}=\bigoplus H^{\bullet}\left(X, S^{\bullet}(\mathbb{Z}) / S^{\bullet+1}(\mathbb{Z})\right)=\bigoplus H^{\bullet}\left(X_{\bullet}, X_{\bullet+1} ; \mathbb{Z}\right)
$$

with appropriate bigradings. Then we have an exact couple of Abelian groups

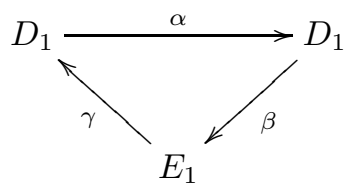

where the morphisms are obtained from the long exact sequence associated to the triple $\left(X, X_{\bullet}, X_{\bullet+1}\right)$. We can define an exact couple in $\mathcal{A}$ with

$$
\begin{gathered}
\mathcal{D}_{1}=\bigoplus \mathcal{H}^{\bullet}\left(X, X_{\bullet}\right) \\
\mathcal{E}_{1}=\bigoplus \mathcal{H}^{\bullet}\left(X_{\bullet}, X_{\bullet+1}\right)
\end{gathered}
$$

in the same way as above. This exact couple maps to the previous one under $\Phi$. The couple yields a spectral sequence $\mathcal{E}_{1}$ in $\mathcal{A}$ which maps to $E_{1}$ under $\Phi$, and converges to $\mathcal{H}^{\bullet}(X)$ with the filtration

$$
\text { image }\left[\mathcal{H}^{i}\left(X, X_{\bullet}\right) \rightarrow \mathcal{H}^{i}(X)\right]
$$

The exact couple, and hence the spectral sequence, is clearly functorial in $\left(X, X_{\bullet}\right)$.

Suppose that $f: X \rightarrow Y$ is a morphism of varieties, and that $Y_{\bullet}$ is a filtration as above. Let $X_{\bullet}=f^{-1} Y_{\bullet}$, then $f:\left(X, X_{\bullet}\right) \rightarrow\left(Y, Y_{\bullet}\right)$ becomes a morphism.

Lemma 3.9. With the above notation and assumptions, $\mathbb{R} f_{*}\left(\mathcal{F}, S\left(X_{\bullet}\right)\right) \cong\left(\mathbb{R} f_{*} \mathcal{F}, S\left(Y_{\bullet}\right)\right)$. for any sheaf $\mathcal{F}$ on $X$.

Proof. Let $X_{a}^{o}=f^{-1} Y_{a}^{o}$, and let $J_{a}: X_{a}^{o} \hookrightarrow X_{a}$ and $I_{a}: X_{a} \rightarrow X K_{a}: X-X_{a} \hookrightarrow X$ denote the inclusions. The lemma follows from the natural equality of sheaves

$$
f_{*} S^{a}\left(X_{\bullet}, \mathcal{I}\right)=k_{a-1 !} f_{*} K_{a-1}^{*} \mathcal{I}=S^{a}\left(Y_{\bullet}, f_{*} \mathcal{I}\right)
$$

for $\mathcal{I}$ on $X$.

Corollary 3.10. There is an isomorphism of spectral sequences

$$
E_{1}^{p q}\left(Y_{\bullet}, \mathbb{R} f_{*} \mathcal{F}\right) \cong E_{1}^{p q}\left(X_{\bullet}, \mathcal{F}\right)
$$

Corollary 3.11. Suppose that $Y_{\bullet}$ is cellular with respect to $R^{b} f_{*} \mathcal{F}$ for all $b$. Then there is an isomorphism of bigraded Abelian groups

$$
E_{2}^{a 0}\left(Y_{\bullet}, R^{b} f_{*} \mathcal{F}\right) \cong E_{2}^{a b}\left(X_{\bullet}, \mathcal{F}\right)
$$

Remark 3.12. This will not be an isomorphism of differential bigraded groups. 
Proof. By the above assumptions, the maps

$$
R^{b} f_{*} \mathcal{F}[-b] \leftarrow \tau_{\leq b} \mathbb{R} f_{*} \mathcal{F} \rightarrow \mathbb{R} f_{*} \mathcal{F}
$$

induce isomorphisms

$$
E_{1}^{a 0}\left(Y_{\bullet}, R^{b} f_{*} \mathcal{F}\right) \cong E_{1}^{p q}\left(Y_{\bullet}, \mathbb{R} f_{*} \mathcal{F}\right)
$$

which will compatible with the differentials.

Lemma 3.13. Let $\left(Y, Y_{\bullet}\right)$ be an object of $F V_{\kappa}, f: X \rightarrow Y$ be a morphism of varieties, and $X_{\bullet}=f^{-1} Y_{\bullet}$. For each sheaf $\mathcal{F}$ on $X$, let

$$
\mathcal{L}_{2}^{p q}(f, \mathcal{F})=H^{p}\left(Y, R^{q} f_{*} \mathcal{F}\right)
$$

denote the Leray spectral sequence. Then there is a natural morphism of spectral sequences

$$
\mathcal{L}_{2}^{p q}(f, \mathcal{F}) \rightarrow E_{2}^{p q}\left(X_{\bullet}, \mathcal{F}\right)
$$

Naturallity means that given objects and morphisms $\left(Y^{\prime}, Y_{\bullet}^{\prime}\right), f^{\prime}: X^{\prime} \rightarrow Y^{\prime}, X_{\bullet}^{\prime}=$ $f^{\prime-1} Y_{\bullet}^{\prime}$, and a commutative diagram

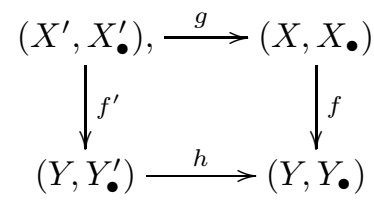

the induced diagram

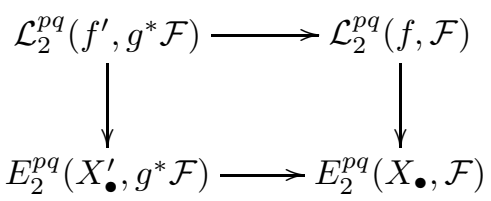

commutes.

Proof. Let $\mathcal{I}^{\bullet}$ be an injective resolution of $\mathcal{F}$. Set $\Sigma^{a}=S^{a}\left(X_{\bullet}, \mathcal{I}^{\bullet}\right)$, then $\left(\mathcal{I}^{\bullet}, \Sigma\right)$ is a filtered acyclic resolution of $\left(\mathcal{F}, S^{\bullet}(X \bullet, \mathcal{F})\right)$ by lemma 3.3 If $G$ denotes the trivial filtration $G^{0}=f_{*} \mathcal{I}$ and $G^{1}=0$, then the inclusion $\left(f_{*} \mathcal{I}, G\right) \rightarrow\left(f_{*} \mathcal{I}, f_{*} \Sigma\right)$ is compatible with the filtrations. Therefore, we get a map of filtered complexes $\left(f_{*} \mathcal{I}, \tau\right) \rightarrow\left(f_{*} \mathcal{I}, \operatorname{Dec}\left(f_{*} \Sigma\right)\right)$, see (5). By definition, there is an exact sequence

$$
0 \rightarrow \operatorname{Dec}(\Sigma)^{p} \mathcal{I}^{n} \rightarrow \Sigma^{p+n} \mathcal{I}^{n} \rightarrow \Sigma^{p+n} \mathcal{I}^{n+1} / \Sigma^{p+n+1} \mathcal{I}^{n+1}
$$

which yields the exact sequence

$$
0 \rightarrow f_{*} \operatorname{Dec}(\Sigma)^{p} \mathcal{I}^{n} \rightarrow f_{*} \Sigma^{p+n} \mathcal{I}^{n} \rightarrow f_{*}\left(\Sigma^{p+n} \mathcal{I}^{n+1} / \Sigma^{p+n+1} \mathcal{I}^{n+1}\right)
$$

Furthermore,

$$
f_{*}\left(\Sigma^{p+n} \mathcal{I}^{n+1} / \Sigma^{p+n+1} \mathcal{I}^{n+1}\right)=f_{*}\left(\Sigma^{p+n} \mathcal{I}^{n+1}\right) / f_{*}\left(\Sigma^{p+n+1} \mathcal{I}^{n+1}\right)
$$

since $\left(\mathcal{I}^{\bullet}, \Sigma\right)$ is a filtered acyclic resolution. Sequences (11) and (12) imply that $\operatorname{Dec}^{\bullet}\left(f_{*} \Sigma\right)=f_{*}(\operatorname{Dec} \bullet \Sigma)$. Therefore, we get a map $\left(f_{*} \mathcal{I}, \tau\right) \rightarrow\left(f_{*} \mathcal{I}, f_{*} \operatorname{Dec}(\Sigma)\right)$, which induces a morphism of spectral sequences

$$
E_{1}^{p q}\left(\mathbb{R} \Gamma\left(f_{*} \mathcal{I}, \tau\right)\right) \rightarrow E_{1}^{p q}\left(\mathbb{R} \Gamma\left(f_{*} \mathcal{I}, f_{*}(\operatorname{Dec} \Sigma)\right)\right)
$$

The spectral sequence on the left is $\mathcal{L}$ up to a shift by (8). While the one on the right coincides with $E_{2}^{p q}\left(X_{\bullet}, \mathcal{F}\right)$ by the same shift thanks to (6) $)$. 
Naturallity follows from lemma 3.9 and the commutativity of the diagram

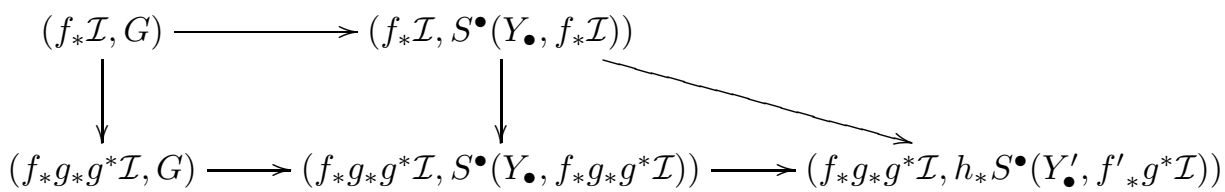

Lemma 3.14. Suppose that $f: X \rightarrow Y$ is a projective morphism in $V_{\kappa}$. Let $h: Y^{\prime} \rightarrow Y$ be a morphism with contractible fibers, and let $f^{\prime}: X^{\prime} \rightarrow Y^{\prime}$ denote the fiber product. Then the Leray spectral sequences

$$
H_{\sigma}^{p}\left(Y, R^{q} f_{\sigma *} \mathbb{Z}\right) \Rightarrow H_{\sigma}^{p+q}(X, \mathbb{Z})
$$

and

$$
H_{\sigma}^{p}\left(Y^{\prime}, R^{q} f_{\sigma *}^{\prime} \mathbb{Z}\right) \Rightarrow H_{\sigma}^{p+q}\left(X^{\prime}, \mathbb{Z}\right)
$$

are isomorphic.

Proof. There is of course, a map of spectral sequences induced by $h^{*}$. It is enough to prove that this is an isomorphism at $E_{2}$. We do this by induction on $\operatorname{dim} Y$. To simplify notation, we will omit $\sigma$ for the remainder of the proof. Let $Y_{1} \subset Y$ be a closed set such that the sheaves $R^{q} f_{*} \mathbb{Z}$ are all locally constant on the complement of $Y_{1}$. Let $Y_{1}^{\prime}=h^{-1} Y_{1}$, and let $i: Y_{1} \rightarrow Y, i^{\prime}: Y_{1}^{\prime} \rightarrow Y^{\prime}, j: Y-Y_{1} \rightarrow Y$ and $j^{\prime}: Y^{\prime}-Y_{1}^{\prime} \rightarrow Y^{\prime}$ denote the inclusions. Then we have the following commutative diagram with exact rows:

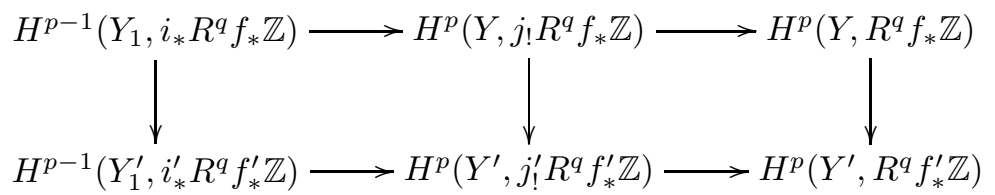

The diagram should be extended two places to the right, but this is omitted for typographic reasons. The second and fifth vertical arrows are isomorphisms because the pairs $\left(Y, Y_{1}\right)$ and $\left(Y^{\prime}, Y_{1}^{\prime}\right)$ with their classical topologies are homotopy equivalent. The first and fourth vertical arrows are isomorphisms by induction. Therefore the third vertical arrow is an isomorphism by the 5 lemma.

Proof of theorem 3.1. Let $f: X \rightarrow Y$ be a projective morphism in $V_{\kappa}$. By lemma 3.14 we can assume that $Y$ is affine. Lemma 3.7 yields a filtration $Y \bullet$ of $Y$ which is cellular with respect to the higher direct image sheaves $R^{q} f_{*} \mathbb{Z}$ (we will suppress $\sigma)$ and satisfies $\operatorname{dim} Y_{i}=i$. Let $X_{\bullet}=f^{-1} Y_{\bullet}$. We wish to show that the map of spectral sequences

$$
\lambda: H^{p}\left(Y, R^{q} f_{*} \mathbb{Z}\right) \rightarrow E_{2}^{p q}\left(X_{\bullet}, \mathbb{Z}\right)
$$

constructed in lemma 3.13 is an isomorphism. It suffices to check that $\lambda$ is an isomorphism at $E_{2}$. Lemma 3.4 and corollary 3.11 yield isomorphisms

$$
H^{p}\left(Y, R^{q} f_{*} \mathbb{Z}\right) \cong E_{2}^{p 0}\left(Y_{\bullet}, R^{q}\right) \cong E_{2}^{p q}\left(X_{\bullet}, \mathbb{Z}\right)
$$


We will be done once we know that the composition of these maps is $\lambda$. From the constructions of these maps, this amounts to the commutativity of the diagram

$$
\begin{array}{cccc}
\left(\mathbb{R} f_{*} \mathbb{Z}, \tau\right) & \rightarrow & \mathbb{R} f_{*}\left(\mathbb{Z}, \operatorname{Dec} S\left(X_{\bullet}\right)\right) \\
\uparrow & & \uparrow \\
\left(\tau_{\leq q} \mathbb{R} f_{*} \mathbb{Z}, \tau\right) & \rightarrow & \left(\tau_{\leq q} \mathbb{R} f_{*} \mathbb{Z}, \operatorname{Dec} S\left(Y_{\bullet}\right)\right) \\
\downarrow & & \downarrow \\
\left(R^{q}[-q], \tau\right) & \rightarrow & \left(R^{q}[-q], \operatorname{Dec} S\left(Y_{\bullet}\right)\right)
\end{array}
$$

This is a straightforward verification.

It follows from lemma 3.8 and the previous paragraph that the Leray spectral sequence can be lifted to $\mathcal{A}$. It remains to check that this lift is independent of $Y_{\bullet}$ and functorial. Suppose that $Y_{\bullet}^{\prime} \subseteq Y$ is another chain which is cellular with respect to the higher direct image sheaves and which satisfies the above dimension conditions. Lemma 3.7 yields a third cellular filtration $Y_{\bullet}^{\prime \prime} \supseteq Y_{\bullet} \cup Y_{\bullet}^{\prime}$. Let $X_{\bullet}^{\prime}=$ $f^{-1} Y_{\bullet}^{\prime}, X_{\bullet}^{\prime \prime}=f^{-1} Y_{\bullet}^{\prime \prime}$ and let $\lambda^{\prime}, \lambda^{\prime \prime}$ be the corresponding isomorphisms. Then we get maps

$$
E_{1}^{p q}\left(X_{\bullet}^{\prime}, \mathbb{Z}\right) \leftarrow E_{1}^{p q}\left(X_{\bullet}^{\prime \prime}, \mathbb{Z}\right) \rightarrow E_{1}^{p q}\left(X_{\bullet}, \mathbb{Z}\right)
$$

compatible with $\lambda, \lambda^{\prime}$ and $\lambda^{\prime \prime}$. Therefore, these are isomorphisms from $E_{2}$ onwards. It follows that the liftings to $\mathcal{A}$ are isomorphic.

Next suppose that

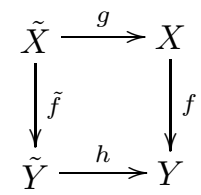

is a commutative diagram of varieties. By corollary 2.4 and lemma 3.14 we can assume that both $Y$ and $\tilde{Y}$ are affine. Let $\tilde{Y} \bullet \subseteq \tilde{Y}$ be a filtration which is cellular with respect to all the $R^{q} \tilde{f}_{*} \mathbb{Z}$ and such that $\operatorname{dim} \tilde{Y}_{i}=i$. By lemma 3.7 we can construct a filtration $Y_{\bullet} \supseteq h\left(\tilde{Y}_{\bullet}\right)$ which is cellular with respect to all $R^{q} f_{*} \mathbb{Z}$. Let $\tilde{X}_{\bullet}=\tilde{f}^{-1} \tilde{Y}_{\bullet}$. Then there is a morphism of spectral sequences

$$
E_{2}^{p q}\left(X_{\bullet}, \mathbb{Z}\right) \rightarrow E_{2}^{p q}\left(\tilde{X}_{\bullet}, \mathbb{Z}\right)
$$

compatible with the map on Leray spectral sequences.

\section{Compactly Supported COHOMOlogy}

In this section, we will sketch a couple of generalizations of theorem 3.1 Once again, fix an embedding $\sigma: \kappa \hookrightarrow \mathbb{C}$ and an enriched $\sigma$-Betti theory $\left(\mathcal{H}: V_{\kappa}^{2} \rightarrow \mathcal{A}, \Phi\right)$. Let $f: X \rightarrow Y$ be a projective morphism of quasiprojective varieties over $\kappa$, and let $\tilde{Y} \subseteq Y$ be closed and $\tilde{X}=f^{-1} \tilde{Y}$. Let $J: X-\tilde{X} \hookrightarrow X$ and $j: Y-\tilde{Y} \hookrightarrow Y$ denote the inclusions. There are isomorphisms $j_{!} R^{q} f_{\sigma, *} \mathbb{Z}=R^{q} f_{\sigma, *} J_{!} \mathbb{Z}$. Thus we can form the Leray spectral sequence for pairs

$$
\mathcal{L}_{2}^{p q}(f, \tilde{Y}, \mathbb{Z})=H_{\sigma}^{p}\left(Y, j_{!} R^{q} f_{\sigma *} \mathbb{Z}\right) \Rightarrow H_{\sigma}^{p+q}(X, \tilde{X}, \mathbb{Z})
$$

This is functorial in the sense that a commutative diagram

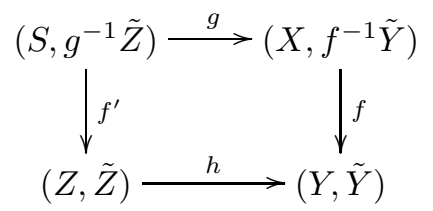


induces morphism

$$
\mathcal{L}_{2}^{p q}(f, \tilde{Y}, \mathbb{Z}) \rightarrow \mathcal{L}_{2}^{p q}\left(f^{\prime}, \tilde{Z}, \mathbb{Z}\right) .
$$

Theorem 4.1. This spectral sequence lifts naturally to $\mathcal{A}$.

The proof is almost identical to the proof of theorem 3.1 but a couple of modifications need to be made. We suppress $\sigma$ as above. We can reduce to the case where $Y$ is affine by lemma 2.3 and the following extension of lemma 3.14 to pairs:

Lemma 4.2. Let $h: Y^{\prime} \rightarrow Y$ be a morphism with contractible fibers. Let $f^{\prime}: X^{\prime} \rightarrow$ $Y^{\prime}$ denote the fiber product and $\tilde{Y}^{\prime}=h^{-1} \tilde{Y}$ etcetera. Then the spectral sequences

$$
H^{p}\left(Y, j_{!} R^{q} f_{*} \mathbb{Z}\right) \Rightarrow H^{p+q}(X, \tilde{X}, \mathbb{Z})
$$

and

are isomorphic.

$$
H^{p}\left(Y^{\prime}, j_{!}^{\prime} R^{q} f_{*}^{\prime} \mathbb{Z}\right) \Rightarrow H^{p+q}\left(X^{\prime}, \tilde{X}^{\prime}, \mathbb{Z}\right)
$$

This extension follows immediately from lemma 3.14 and the exact sequence for a pair. We then construct a cellular filtration $Y_{\bullet}$ with respect to the sheaves $R^{q} f_{*} J_{!} \mathbb{Z}=j_{!} R^{q} f_{*} \mathbb{Z}$. Arguing as above, we see that (13) is isomorphic to $E_{2}^{p q}\left(f^{-1} Y_{\bullet}, J_{!} \mathbb{Z}\right)$. The remaining ingredient is the following extension of lemma 3.8

Lemma 4.3. For any pair $\left(X, X_{\bullet}\right)$ in $F V_{\kappa}$ with $J: X-\tilde{X} \rightarrow X$ as above,

$$
E_{1}^{p q}\left(X_{\bullet}, J_{!} \mathbb{Z}\right) \Rightarrow H^{p+q}\left(X, J_{!} \mathbb{Z}\right)
$$

can be lifted naturally to $\mathcal{A}$.

In fact, it is possible to use the original form of the lemma by choosing $\tilde{Y}$ as one of the members of the cellular filtration; this is possible since the sheaves $R^{q} f_{*} J_{!} \mathbb{Z}$ vanish along $\tilde{Y}$. The argument for independence from $Y_{\bullet}$ and functoriality are essentially as before.

Corollary 4.4. Assume $\kappa=\mathbb{C}$. Let $f: X \rightarrow Y$ be a projective map of quasiprojective varieties. Then the Leray spectral sequence for compactly supported cohomology

$$
\mathcal{L}_{c, 2}^{p q}=H_{c}^{p}\left(Y, R^{q} f_{*} \mathbb{Z}\right) \Rightarrow H_{c}^{p+q}(X, \mathbb{Z})
$$

is compatible with mixed Hodge structures.

Proof. Choose projective compactifications $\bar{X}$ and $\bar{Y}$. By blowing up $\bar{X}$ along $\bar{X}-X$ we can assume that $f$ extends to a morphism of the compactifications. Apply the theorem to the map of pairs $(\bar{X}, \bar{X}-X) \rightarrow(\bar{Y}, \bar{Y}-Y)$.

If $Y$ is affine we can give another proof of this corollary.

Second proof for $Y$ affine. Proceed as in the proof theorem 3.1 and construct a cellular decomposition $Y_{\bullet}$ with respect to $R^{q} f_{*} \mathbb{Z}$, but now replace all occurrences of ordinary cohomology with compactly support cohomology. In particular, the above spectral sequence will become isomorphic to

$$
E_{1}=H_{c}^{p+q}\left(X_{p}, X_{p+1}, \mathbb{Z}\right) \Rightarrow H_{c}^{p+q}(X, \mathbb{Z})
$$

from $E_{2}$ onwards, where $X_{p}=f^{-1} Y_{p}$.

The second proof leads to an estimate on weights analogous to the $l$-adic situation D3. Let us say that a mixed Hodge structure $H$ has weights $\leq k$ if $G r_{i}^{W} H=0$ for $i>k$. 
Theorem 4.5. If $f: X \rightarrow Y$ is a projective map of complex quasiprojective varieties, then $H_{c}^{i}\left(Y, R^{j} f_{*} \mathbb{Z}\right)$ has weights $\leq i+j$.

Proof. Suppose that $Y$ is affine, then from the previous proof $H_{c}^{i}\left(Y, R^{j} f_{*} \mathbb{Z}\right)$ is a subquotient of $H_{c}^{i+j}\left(X_{j}, X_{j+1}, \mathbb{Z}\right)$, and this has weights $\leq i+j$ by [D2, 8.2.4] and the exact sequence of the pair.

In general, choose an affine open set $U \subset Y$, and let $Z=Y-U$. Then the theorem follows from induction on $\operatorname{dim} Y$ and the exact sequence

$$
H_{c}^{i}\left(U, R^{j} f_{*} \mathbb{Z}\right) \rightarrow H_{c}^{i}\left(Y, R^{j} f_{*} \mathbb{Z}\right) \rightarrow H_{c}^{i}\left(Z, R^{j} f_{*} \mathbb{Z}\right)
$$

Remark 4.6. This proof yields something stronger. Namely, that the $(p, q)$, for which the $(p, q)$ th Hodge number of $H_{c}^{i}\left(Y, R^{j} f_{*} \mathbb{Z}\right)$ is nonzero, lie within the shaded triangle of Figure 1.

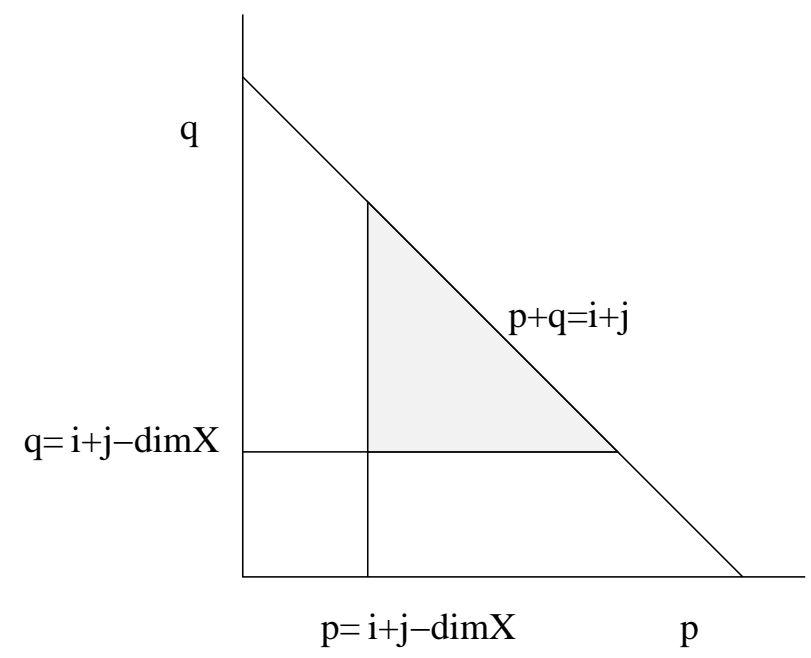

FIGURE 1.

\section{Geometric Variations of Hodge Structure}

All varieties in this section will be defined over $\mathbb{C}$. For the record, we will briefly recall the definition of variation of Hodge structure; see [BZ, and reference contained there, for a more detailed discussion. A rational polarizable variation of Hodge structure of weight $k$ on a smooth algebraic variety $Y$ consists of

(1) a local system or locally constant sheaf of $\mathbb{Q}$ vector spaces $V$ on $Y$.

(2) a filtration by subbundles $F^{\bullet} \subset V \otimes O_{X}$.

We require that $F^{\bullet}$ should satisfy Griffiths transversality and that the data induce a pure Hodge structure of weight $k$ on the fibers. We also require that the whole thing admits a flat polarization (which will not be fixed). When no confusion is likely, we use the same symbol for the variation of Hodge structure and its local system. A morphism is a homomorphism of local systems which preserves filtrations. Let $\mathrm{PVSH}_{k}(Y)$ be the additive (and in fact Abelian) category of polarizable variations 
of Hodge structure over $Y$. The motivating examples come from geometry. If $f: X \rightarrow Y$ is a smooth projective map of varieties then $R^{k} f_{*} \mathbb{Q}$ is part of a polarizable variation of Hodge structure of weight $i$ with

$$
F^{p}=\text { image }\left[\mathbb{R}^{k} \Omega_{X / Y}^{\geq p} \rightarrow \mathbb{R}^{k} \Omega_{X / Y}^{\bullet} \cong R^{k} f_{*} \mathbb{Q} \otimes O_{Y}\right]
$$

We define a variation of Hodge structure to be geometric if is isomorphic to a direct summand of some $R^{i} f_{*} \mathbb{Q}$, with $f$ smooth and projective. Let $\operatorname{GVSH}_{k}(Y)$ be the full subcategory of geometric variations of Hodge structure. Given a morphism $g: Y^{\prime} \rightarrow Y$, there is an exact functor $g^{*}: \operatorname{PVSH}(Y) \rightarrow \operatorname{PVSH}\left(Y^{\prime}\right)$ defined by $V \mapsto g^{*} V, F^{\bullet} \mapsto g^{*} F^{\bullet} . g^{*}$ preserves $\mathrm{GVSH}_{k}$, since $g^{*} R^{k} f_{*} \mathbb{Q}=R^{k} f_{*}^{\prime} \mathbb{Q}$ where $f^{\prime}$ is obtained by base change.

Theorem 5.1. For each smooth quasiprojective variety $T$, there are functors $H_{M H S}^{i}$ : $G V_{V S H}(T) \rightarrow \mathbb{Q}$-MHS such that:

(1) The rational lattice for $H_{M H S}^{i}(T, V)$ is just ordinary cohomology of the local system of $V$.

(2) Let $g: T^{\prime} \rightarrow T$ be a morphism of varieties and let $V \in G V S H(T)$, there is a morphism $g^{*}: H_{M H S}^{i}(T, V) \rightarrow H_{M H S}^{i}\left(T^{\prime}, g^{*} V\right)$ compatible with the usual pullback on cohomology.

(3) If $f: X \rightarrow T$ is a smooth projective map, then $H_{M H S}^{p}\left(T, R^{q} f_{*} \mathbb{Q}\right)$ is a subquotient of the canonical mixed Hodge structure on $H^{p+q}(X, \mathbb{Q})$.

The proof will be based on a series of lemmas.

Lemma 5.2. If $F: Z \rightarrow T$ is a smooth projective morphism, then

$$
L^{p} H^{i}(Z, \mathbb{Q}) \cup L^{q} H^{j}(Z, \mathbb{Q}) \subseteq L^{p+q} H^{i+j}(Z, \mathbb{Q})
$$

Proof. Let $Z_{p}=F^{-1} T_{p}$ be as in theorem 2.1. Then the lemma follows from the commutativity of the diagram

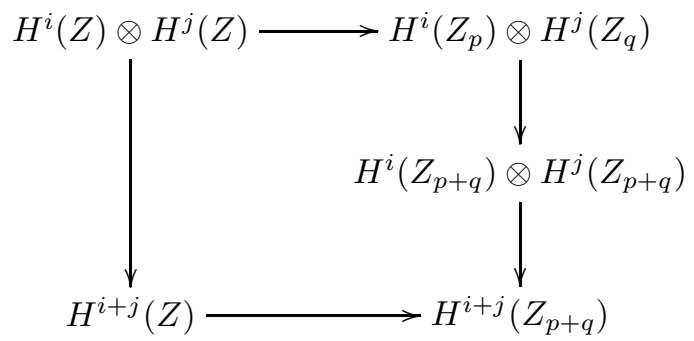

Given a proper map $q: Z \rightarrow Y$ of smooth varieties. The pushforward map $q_{*}$ : $H^{*}(Z) \rightarrow H^{*}(Y)$ is Poincaré dual to the pullback map $q^{*}$ on compactly supported cohomology. This is a morphism of mixed Hodge structure.

Lemma 5.3. Suppose that

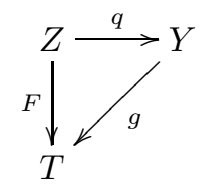


is a commutative diagram of smooth projective maps between smooth quasiprojective varieties. Then

$$
q_{*}\left(L^{p} H^{i}(Z, \mathbb{Q})\right) \subseteq L^{p} H^{i-2 d}(Y, \mathbb{Q})
$$

where $d=\operatorname{dim} Z-\operatorname{dim} Y$.

Proof. Let $Y_{p}=g^{-1} T_{p}$ and $Z_{p}=F^{-1} T_{p}=q^{-1} Y_{p}$ in the notation of theorem 2.1. We have a commutative diagram

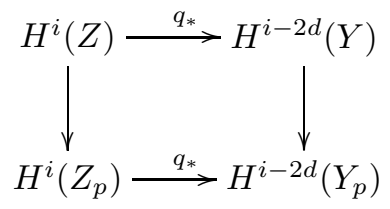

The lemma follows from this and theorem 2.1

Let $f: X \rightarrow T$ and $g: Y \rightarrow T$ be smooth projective maps. Let us write $f \times g$ : $X \times_{T} Y \rightarrow T$ for the fiber product, and $p_{1}: X \times_{T} Y \rightarrow X$ and $p_{2}: X \times_{T} Y \rightarrow Y$ for the projections. Suppose that the fiber dimension of $f$ is $d$. Given a morphism $\gamma: R^{i} f_{*} \mathbb{Q} \rightarrow R^{i} g_{*} \mathbb{Q}$, the class of its graph $[\gamma]$ is the image of $\gamma$ under

$$
\begin{aligned}
\operatorname{Hom}_{V H S}\left(R^{i} f_{*} \mathbb{Q}, R^{i} g_{*} \mathbb{Q}\right) & \cong \operatorname{Hom}_{V H S}\left(\mathbb{Q}(0), R^{2 d-i} f_{*} \mathbb{Q}(d) \otimes R^{i} g_{*} \mathbb{Q}\right) \\
& \subseteq \operatorname{Hom}_{V H S}\left(\mathbb{Q}(0), R^{2 d}(f \times g)_{*} \mathbb{Q}(d)\right)
\end{aligned}
$$

Thus $[\gamma]$ can be viewed as a constant degree $2 d$ Hodge cycle, i.e. rational $(d, d)$ cycle, along the fibers of $f \times g$.

Lemma 5.4. With the above notation, $\gamma$ coincides with the composite of

$$
R^{i} f_{*} \mathbb{Q} \stackrel{p_{1}^{*}}{\longrightarrow} R^{i}(f \times g)_{*} \mathbb{Q} \stackrel{\cup[\gamma]}{\longrightarrow} R^{i+2 d}(f \times g)_{*} \mathbb{Q}(d) \stackrel{p_{2 *}}{\longrightarrow} R^{i} f_{*} \mathbb{Q}
$$

Proof. It is enough to check this at the stalk level, and this is well known $\mathrm{K}$, 1.3.7].

Proof of theorem [5.1] By [D1, there is an isomorphism of vector spaces

$$
H^{p}\left(T, R^{q} f_{*} \mathbb{Q}\right) \cong G r_{L}^{p} H^{p+q}(X, \mathbb{Q})
$$

Corollary 2.6 implies that the right hand side carries a mixed Hodge structure. We use this isomorphism to define the mixed Hodge structure on the left. Thus (3) is true by decree. More generally, suppose that $V$ is a variation of Hodge structure which is a direct summand of $R^{i} f_{*} \mathbb{Q}$. Then $V$ is the image of a projection $P: R^{i} f_{*} \mathbb{Q} \rightarrow R^{i} f \mathbb{Q}$. By the theorem of the fixed part [D2, 4.1.1], the graph

$$
[P] \in \operatorname{Hom}_{V H S}\left(\mathbb{Q}(0), R^{2 d}(f \times f)_{*} \mathbb{Q}(d)\right)
$$

can be lifted to a Hodge cycle $\phi \in H^{2 d}(X, \mathbb{Q})$. The map $\Phi(\alpha)=p_{2 *}\left(p_{1}^{*} \alpha \cup \phi\right)$ induces a morphism $\Phi: H^{i+j}(X, \mathbb{Q}) \rightarrow H^{i+j}(X, \mathbb{Q})$ of mixed Hodge structures, which by (3) and lemmas 5.2 and 5.3 preserves the Leray filtrations. By lemma 5.4

$$
G r_{L}^{i}(\Phi): H^{j}\left(X, R^{i} f_{*} \mathbb{Q}\right) \rightarrow H^{j}\left(X, R^{i} f_{*} \mathbb{Q}\right)
$$

coincides with the map induced by $P$. This shows that $P$ is a morphism of mixed Hodge structures. Therefore we can equip $H^{j}(X, V)$ with the mixed Hodge structure on the image $P\left(H^{j}\left(T, R^{i} f_{*} \mathbb{Q}\right)\right)$.

We have to verify that the mixed Hodge structure just constructed on $H^{j}(X, V)$ is independent of choices. Suppose that $V$ is a direct summand of both $R^{i} f_{*} \mathbb{Q}$ and 
$R^{i} g_{*} \mathbb{Q}$ for two smooth projective maps $f: X \rightarrow T$ and $g: Y \rightarrow T$. Then consider the commutative diagram

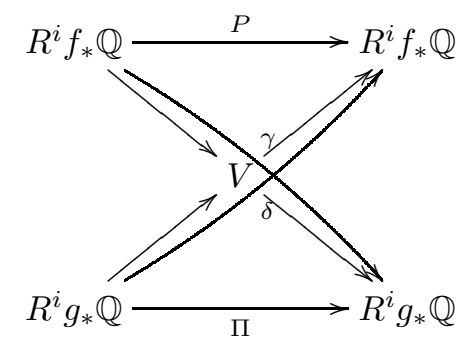

where all the unlabeled arrows are the projections and inclusions to and from $V$. $\gamma(\delta)$ is the arrow connecting the upper (respectively lower) left and lower (respectively upper) right corners. The relations $\gamma P=\gamma$ and $\gamma \delta=\Pi$ are easily verified. We get an induced diagram

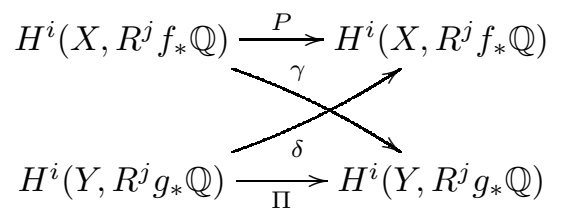

We can see that these maps are morphisms of mixed Hodge structures by expressing them as compositions of pullbacks, pushforwards and cup products with Hodge cycles as above. The relations $\gamma P=\gamma$ and $\gamma \delta=\Pi$ imply that $\gamma$ induces a surjection $\operatorname{image}(P) \rightarrow \operatorname{image}(\gamma)$ and that there is an inclusion $\operatorname{image}(\Pi) \subseteq \operatorname{image}(\gamma)$. Since

$$
\operatorname{dim} \operatorname{image}(P)=\operatorname{dim} \operatorname{image}(\Pi)=\operatorname{dim} H^{i}(X, V),
$$

it follows that image $(P)$ and image $(\Pi)$ are isomorphic as mixed Hodge structures.

Finally property (2) is clear from construction.

Corollary 5.5. Let $f: X \rightarrow T$ be a smooth projective map of smooth quasiprojective varieties, then $H^{j}\left(T, S^{N}\left(R^{i} f_{*} \mathbb{Q}\right)\right)$ carries a natural mixed Hodge structure for each positive integer $N$. The same holds if the symmetric power is replaced by an exterior power or, more generally, a Schur functor.

Proof. These are geometric variations of Hodge structure, since they are direct summands of $R^{i}(f \times f \times \ldots f)_{*} \mathbb{Q}$.

\section{ThrEeFolds FIBERED BY RATIONAL CURVES}

We end this paper with a geometric example. The details are sketched. Let $X$ be a smooth projective threefold over $\mathbb{C}$ with a flat map $f: X \rightarrow S$ such that the fibers are connected rational curves. Let $C$ be the discriminant set. Our goal is to compute the intermediate Jacobian

$$
J^{2}(X)=\frac{H^{3}(X, \mathbb{C})}{F^{2} H^{3}(X, \mathbb{C})+H^{3}(X, \mathbb{Z})}
$$

generalizing the usual description for conic bundles [CG]. This amounts to computing the Hodge structure on $H^{3}(X)$ modulo torsion. 
We start by computing higher direct images. The fibers of $f$ are rational curves which are homotopic to a wedge of spheres. Therefore $f_{*} \mathbb{Z}=\mathbb{Z}$,

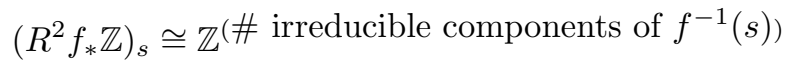

and all other $R^{i} f_{*} \mathbb{Z}=0$. Thus the Leray spectral sequence (3.2) yields an exact sequence of Hodge structures

$$
0 \rightarrow \mathcal{L}_{\infty}^{30} \rightarrow H^{3}(X, \mathbb{Z}) \rightarrow \mathcal{L}_{\infty}^{12} \rightarrow 0
$$

We certainly have $\mathcal{L}_{2}^{30}=\mathcal{L}_{3}^{30}$ and $\mathcal{L}_{2}^{12}=\mathcal{L}_{3}^{12}$. Consider the differentials

$$
\begin{aligned}
& d_{3}: \mathcal{L}_{3}^{02} \rightarrow \mathcal{L}_{3}^{30} \\
& d_{3}: \mathcal{L}_{3}^{12} \rightarrow \mathcal{L}_{3}^{40}
\end{aligned}
$$

The right sides are pure of weights 3 and 4 respectively, while the left sides have smaller weights by theorem 4.5 Therefore these differentials vanish. (This can also be deduced by observing that the maps $H^{i}(S, \mathbb{Z}) \rightarrow H^{i}(X, \mathbb{Z})$ are injective modulo torsion because of Poincaré duality.) Thus $\mathcal{L}_{\infty}^{30}=H^{3}(S, \mathbb{Z})$ and $\mathcal{L}_{\infty}^{12}=$ $H^{1}\left(S, R^{2} f_{*} \mathbb{Z}\right)$. The sequence (14) leads an exact sequence of tori

$$
0 \rightarrow \operatorname{Alb}(S) \rightarrow J^{2}(X) \rightarrow J^{2} H^{1}\left(S, R^{2} f_{*} \mathbb{Z}\right) \rightarrow 0
$$

To proceed further, let us make the simplifying assumptions that $A l b(S)=0$ or equivalently that $H^{1}(S, \mathbb{Z})$ is torsion, that $C$ is smooth, and that $f^{-1} C \rightarrow C$ is topologically locally trivial. Choose an embedding

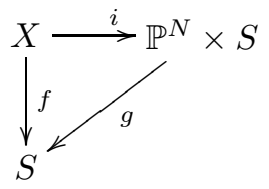

Then $R^{2} g_{*} \mathbb{Z} \cong \mathbb{Z}_{S}$ injects into $R^{2} f_{*} \mathbb{Z}$. The torsion free part $L$ of $R^{2} f_{*} \mathbb{Z} / R^{2} g_{*} \mathbb{Z}$ is supported on $C$. From the long exact sequence, we obtain an isomorphism

$$
H^{1}\left(S, R^{2} f_{*} \mathbb{Z}\right) \cong H^{1}(C, L)
$$

Consider the diagram

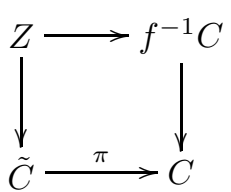

where the $Z \rightarrow \tilde{C}$ is the Stein factorization of normalization of $f^{-1} C . L \otimes \mathbb{Q}$ is a quotient of $\pi_{*} \mathbb{Q}$. It follows easily that $J^{2}(X)$ is isogenous to a quotient of the Jacobian $J(\tilde{C})$. In the case of conic bundle, $L$ is the anti-invariant part of $\pi_{*} \mathbb{Z}$ under the involution of the double cover $\tilde{C} \rightarrow C$. So we recover the usual description of $J^{2}(X)$ as a Prym variety. 


\section{REFERENCES}

[AS] D. Arapura, P. Sastry, Hodge structures and intermediate Jacobians of moduli spaces, Proc. Ind. Acad. Sci 110 (2000)

[BBD] A. Beilinson, J. Bernstein, P. Deligne, Faiseaux Perverse, Asterisque 100 (1982)

[BZ] J. L. Brylinski, S. Zucker, An overview of recent advances in Hodge theory, Sev. Complex Variables VI, Springer-Verlag (1990)

[CG] C. H. Clemens, P. Griffiths The Intermediate Jacobian of the Cubic Threefold Ann. Math. $95(1972)$

[D1] P. Deligne, Théorème de Lefschetz et critéres de dégénérescence de suites spectrales Publ. IHES 35 (1968)

[D2] P. Deligne, Théorie de Hodge II, III Publ. IHES 40, 44, (1971, 1974)

[D3] P. Deligne, La conjecture de Weil II, Publ. IHES 52 (1980)

$[\mathrm{H}] \quad \mathrm{R}$. Hain, Torelli groups and geometry of moduli spaces of curves. Current topics in complex geometry, MSRI Publ. (1995)

[HL] H. Hamm, D.T. Le, Vanishing theorems for constructible sheaves. II. Kodai Math. J. 21 (1998)

[GM] M. Goresky, R. Macpherson, Intersection homology II, Invent. Math 72 (1983)

[J] U. Jannsen, Mixed motives and algebraic K-theory, Lect notes in math 1400, Springer-Verlag (1990)

[Jo] J.P. Jouanolou, Un suit exacte de Mayer-Vietoris en K-theorie algebrique,in Algebraic Ktheory, Lect. Notes Math 341, Springer-Verlag (1973)

[K] S. Kleiman, Algebraic cycles and the Weil conjectures, Dix Esposes sur la cohomologies les schemas, North Holland (1968)

[L] J. Lewis, A filtration on the Chow groups of a complex projective variety, Compositio Math. $128(2001)$

[N] M. Nori, Constructible sheaves, Proc. Int. Conf. on Algebra..., TIFR (2002)

[P] C. Peters, Letter to the author, May 26, 2004

[S1] M. Saito, Modules Hodge polarizables Publ. RIMS 24 (1988)

[S2] M. Saito, Mixed Hodge modules Publ. RIMS 26 (1990)

[W] C. Weibel, An introduction to homological Algebra, Cambridge U. Press (1994)

[Z] S. Zucker, Hodge theory with degenerating coefficients..., Annals of Math. 109 (1979)

Department of Mathematics, Purdue University, West Lafayette, IN 47907, U.S.A.

E-mail address: dvb@math.purdue.edu 